\title{
Cryptotanshinone attenuates isoprenaline-induced cardiac fibrosis in mice associated with upregulation and activation of matrix metalloproteinase-2
}

\author{
SHUANGTAO MA*, DACHUN YANG* ${ }^{*}$ KUIYING WANG, BING TANG, DE LI and YONGJIAN YANG
}

Department of Cardiology, General Hospital of PLA Chengdu Military Area Command, Chengdu, Sichuan 610083, P.R. China

Received December 28, 2011; Accepted April 4, 2012

DOI: $10.3892 / \mathrm{mmr} .2012 .866$

\begin{abstract}
Cryptotanshinone is an active ingredient of Salvia miltiorrhiza that has been used in traditional Chinese medicine for treating cardiovascular disorders. Thus, we investigated the effects of cryptotanshinone on cardiac fibrosis induced by isoprenaline and examined whether cardiac matrix metalloproteinase (MMP)-2 is involved in this process. Male C57BL/6 mice received a daily injection of $0.9 \%$ saline, $3 \mathrm{mg} / \mathrm{kg}$ isoprenaline or isoprenaline plus $20 \mathrm{mg} / \mathrm{kg}$ cryptotanshinone by gastric gavage for 2 weeks. In this study we demonstrated that cryptotanshinone was able to significantly ameliorate isoprenaline-induced cardiac fibrosis, which was associated with a marked upregulation and activation of MMP-2 in the ventricular myocardium. Additionally, we demonstrated that cryptotanshinone dose-dependently upregulated and activated MMP-2 in cultured cardiac fibroblasts. Moreover, incubation with cryptotanshinone also prevented the isoprenaline-induced downregulation and inactivation of MMP-2 in cultured cardiac fibroblasts. Taken together, our data suggest that cryptotanshinone is a novel and potent antifibrotic agent. The present findings further our understanding of the role of MMP-2 in cardiac fibrosis and the antifibrotic mechanisms of cryptotanshinone.
\end{abstract}

\section{Introduction}

Progressive cardiac fibrosis is involved in various processes of cardiovascular diseases. It induces cardiac dysfunction, including myocardial stiffening, and results in reduced myocardial contractility and, ultimately, heart failure (1-3). The attenuation of cardiac fibrosis has been viewed as a novel heart-failure treatment target (4). However, there is currently

Correspondence to: Dr Yongjian Yang, Department of Cardiology, General Hospital of PLA Chengdu Military Area Command, Tianhui, Jinniu, Chengdu, Sichuan 610083, P.R. China

E-mail: yongjiany@yahoo.cn

${ }^{*}$ Contributed equally

Key words: cryptotanshinone, matrix metalloproteinase-2, cardiac fibrosis no effective strategy for the treatment and/or prevention of cardiac fibrosis (5).

The accumulation of extracellular matrix (ECM), which characterizes cardiac fibrosis, is due to the reduced degeneration of collagen (6). The principal enzyme system which is primarily responsible for ECM turnover involves the matrix metalloproteinases (MMPs), specifically MMP-2 (7,8). Indeed, the inappropriate reduction of MMP-2 activity associated with the deposition of collagen has been observed in the development of fibrosis in diabetic cardiomyopathy $(9,10)$. Therefore, therapeutic interventions aimed at the activation of MMP-2 may be considered as one of the most effective strategies for the management of cardiac fibrosis (11).

The Chinese herb Salvia miltiorrhiza, which has beneficial effects on the circulatory system, has been widely used in traditional oriental medicine for the treatment of patients with cardiovascular disorders $(12,13)$. In particular, tanshinone, one of the active components of Salvia miltiorrhiza, has been reported to have antihypertrophic and antifibrotic effects in cultured cardiac myocytes and cardiac fibroblasts $(14,15)$. Cryptotanshinone, another active compound extracted from Salvia miltiorrhiza $(16,17)$, has also been involved in the treatment of cardiovascular diseases. However, there have been no studies performed concerning the possible antifibrotic effect of cryptotanshinone.

The present study aimed to determine whether isoprenaline reduces the protein expression and activity of MMP-2 in cultured cardiac fibroblasts and rat myocardium and whether cryptotanshinone is able to normalize MMP-2 expression and activity and attenuate cardiac fibrosis induced by isoprenaline.

\section{Materials and methods}

Animals. Male C57BL/6 mice 6-8 weeks of age were obtained from our local animal center. The mice were housed under a 12-h/12-h day/night cycle, with ad libitum food and water. The experimental procedures were approved by the Institutional Animal Care and Use Committee of the General Hospital of PLA Chengdu Military Area Command (Chengdu, China). The mice were randomly assigned to one of the following groups: i) control (Con, $n=8)$, which received a daily injection of $0.9 \%$ saline s.c.; ii) isoprenaline (ISO, $\mathrm{n}=8$ ), which received $3 \mathrm{mg} / \mathrm{kg}$ isoprenaline s.c. once daily; iii) isoprenaline plus 
Table I. Morphological parameters of the mice.

\begin{tabular}{lccccc}
\hline Groups & $\mathrm{BW}(\mathrm{g})$ & $\mathrm{HW}(\mathrm{g})$ & $\mathrm{LVW}(\mathrm{g})$ & $\mathrm{HW} / \mathrm{BW}(\mathrm{g} / \mathrm{kg})$ & $\mathrm{LVW} / \mathrm{BW}(\mathrm{g} / \mathrm{kg})$ \\
\hline Control & $30.5 \pm 0.96$ & $0.115 \pm 0.003$ & $0.102 \pm 0.003$ & $3.76 \pm 0.02$ & $3.33 \pm 0.04$ \\
ISO & $31.8 \pm 1.04$ & $0.136 \pm 0.005^{\mathrm{b}}$ & $0.121 \pm 0.005^{\mathrm{b}}$ & $4.26 \pm 0.03^{\mathrm{b}}$ & $3.79 \pm 0.03^{\mathrm{b}}$ \\
ISO+CTS & $31.1 \pm 1.04$ & $0.122 \pm 0.004^{\mathrm{c}}$ & $0.110 \pm 0.003^{\mathrm{c}}$ & $3.94 \pm 0.03^{\mathrm{d}}$ & $3.53 \pm 0.05^{\mathrm{d}}$ \\
\hline
\end{tabular}

Data are presented as mean \pm SEM values of 8 mice per group. BW, body weight; HW, heart weight; LVW, left ventricular weight; HW/BW, heart weight to body weight ratio; LVW/BW, left ventricular weight to body weight ratio; ISO, isoprenaline; CTS, cryptotanshinone. ${ }^{a} \mathrm{P}<0.05$, ${ }^{\mathrm{b}} \mathrm{P}<0.01$ vs. control group; ${ }^{\mathrm{c}} \mathrm{P}<0.05,{ }^{\mathrm{d}} \mathrm{P}<0.01$ vs. ISO group.

cryptotanshinone (ISO+CTS, $\mathrm{n}=8$ ), which received $20 \mathrm{mg} / \mathrm{kg} /$ day cryptotanshinone (gastric gavage) plus the daily injection of isoprenaline. The amount of cryptotanshinone administered was the same as that in a previous study (18).

Hemodynamic measurement. Following 2 weeks of treatment, all the mice were anesthetized with urethane $(1.2 \mathrm{~g} / \mathrm{kg}$, i.p.). The right carotid artery was cannulated with a polyethylene tube and forwarded to the left ventricle. The tube was connected to a pressure transducer. The left ventricular pressure was recorded in synchrony with the RM-6000 polygraph system (Nihon Kohden, Tokyo, Japan). The left ventricular end-systolic pressure (LVESP), left ventricular end-diastolic pressure (LVEDP) and left ventricular $\mathrm{dP} / \mathrm{dt} \max / \mathrm{min}$ were calculated.

Heart weight. At the end of treatment, the animals were sacrificed under deep anesthesia with urethane and the hearts were removed. The ventricles were blotted and weighed and the whole heart weight/body weight (HW/BW) and left ventricular weight $(\mathrm{LVW}) / \mathrm{BW}$ were calculated. The left ventricular tissue was dissected and frozen at $-70^{\circ} \mathrm{C}$ for western blotting and gelatin zymography.

Histological determination of fibrosis. Paraffin-embedded left ventricular tissue was cut into $5-\mu \mathrm{m}$ sections which were stained with picrosirius red and assessed using standard light microscopy. The correlation between the red-stained and the total area of the whole heart section was analyzed as previously described (19).

Cardiac fibroblast culture. Primary adult mouse cardiac fibroblasts were isolated by proteolytic dissociation of the ventricular tissue and cultured using standard protocols as previously described (20). The ventricles were cut into sections and incubated for $10 \mathrm{~min}$ in $\mathrm{KB}$ buffer, consisting of $70 \mathrm{mM}$ $\mathrm{KCl}, 30 \mathrm{mM} \mathrm{K} \mathrm{HPO}_{4}, 5 \mathrm{mM} \mathrm{MgSO}$, $0.5 \mathrm{mM}$ EGTA, $22 \mathrm{mM}$ glucose, $20 \mathrm{mM}$ taurine, $5 \mathrm{mM}$ creatine, $10 \mathrm{mM}$ succinic acid, $2 \mathrm{mM}$ pyruvic acid, $5 \mathrm{mM}$ ATP, $2 \mathrm{mM}$ butyric acid and $115 \mathrm{U} /$ mg collagenase ( $\mathrm{pH} 7.4$ ). The cell suspension was filtered through a $250-\mu \mathrm{m}$ nylon mesh and centrifuged at $25 \mathrm{x}$ g for $3 \mathrm{~min}$. The supernatant was centrifuged at $250 \mathrm{x} \mathrm{g}$ for $10 \mathrm{~min}$. The cells were resuspended in full growth medium [Dulbecco's modified Eagle's medium (Gibco, Grand Island, NY, USA) supplemented with $10 \%$ FCS, 2 mM L-glutamine, $100 \mu \mathrm{g} / \mathrm{ml}$ penicillin $\mathrm{G}, 100 \mu \mathrm{g} / \mathrm{ml}$ streptomycin and $100 \mathrm{U} / \mathrm{ml}$ penicillin]. Four 1 hour later, the nonattached cells were removed. The cells were grown for 4-5 days in full growth medium and observed using light microscopy. The culture contained $>95 \%$ fibroblasts. Cardiac fibroblasts obtained during early passages were used. One day prior to the experiments, the cells were serum-starved. For one experiment, the fibroblasts were incubated with $0,3,10$ or $30 \mu \mathrm{M}$ cryptotanshinone for $24 \mathrm{~h}$. For another experiment, the fibroblasts were treated with vehicle, $10 \mu \mathrm{M}$ isoprenaline or isoprenaline plus $30 \mu \mathrm{M}$ cryptotanshinone for $24 \mathrm{~h}$.

Western blotting. Western blot analysis was performed as previously reported (21). Briefly, protein lysates were obtained by homogenizing myocardial tissues or cultured cardiac fibroblasts with lysis buffer containing $1 \%$ Triton X-100, $150 \mathrm{mM}$ $\mathrm{NaCl}, 1 \mathrm{mM}$ EDTA, $2.5 \mathrm{mM}$ sodium pyrophosphate, $1 \mathrm{mM}$ $\beta$-glycerophosphate, $1 \mathrm{mM} \mathrm{Na} \mathrm{VO}_{4}, 1 \mu \mathrm{g} / \mathrm{ml}$ leupeptin, $1 \mu \mathrm{g} /$ $\mathrm{ml}$ aprotinin and $20 \mathrm{mM}$ Tris $(\mathrm{pH}$ 7.5). The protein concentration was determined using Bio-Rad protein assay reagent (Bio-Rad, Hercules, CA, USA). Equal amounts of protein from the heart extracts were separated by SDS-PAGE (12\%). The samples were then electroblotted onto a nitrocellulose membrane (Boehringer Mannheim Corporation, Indianapolis, IN, USA) and probed with antibody against MMP-2 (1:500; Sigma-Aldrich Co., St. Louis, MO, USA). After washing, the membrane was incubated with a horseradish peroxidase-conjugated secondary antibody (1:1000; Santa Cruz Biotechnology, Santa Cruz, CA, USA) and bound antibody was visualized using a colored reaction. The relative band densities were quantified by densitometry using the Multi-Analyst software package (Bio-Rad). Equal loading of protein was confirmed by measuring $\beta$-actin expression.

Gelatin zymography. The gelatin-degrading activity of MMP-2 in the left ventricular samples and cultured cardiac fibroblasts was identified by zymography using an assay kit (APPLYGEN, Beijing, China), as described previously (22). Briefly, samples were mixed with sample buffer and separated on $7.5 \%$ SDS-polyacrylamide gels containing $1 \mathrm{mg} / \mathrm{ml}$ of gelatin. Following the removal of SDS, the gel was incubated in buffer overnight and then stained with $0.25 \%$ Coomassie brilliant blue G-250. The gelatinolytic activity was detected and quantified using a GS-800 calibrated densitometer (Bio-Rad).

Statistical analysis. Data are presented as the means \pm SEM. The comparisons between groups were determined using one-way ANOVA with Student's t-test post hoc test (SPSS Inc., Chicago, IL, USA). $\mathrm{P}<0.05$ was considered to indicate a statistically significant result. 
Table II. Hemodynamic parameters of the mice.

\begin{tabular}{lcccc}
\hline Groups & LVEDP $(\mathrm{mmHg})$ & $\operatorname{LVESP}(\mathrm{mmHg})$ & $\mathrm{dP} / \mathrm{dt} \max (\mathrm{mmHg} / \mathrm{sec})$ & $\mathrm{dP} / \mathrm{dt} \mathrm{min}(\mathrm{mmHg} / \mathrm{sec})$ \\
\hline Control & $3.2 \pm 0.3$ & $86 \pm 6$ & $5683 \pm 110$ & $4478 \pm 88$ \\
ISO & $6.3 \pm 0.4^{\mathrm{b}}$ & $100 \pm 4$ & $5471 \pm 110$ & $4153 \pm 64^{\mathrm{a}}$ \\
ISO+CTS & $5.2 \pm 0.3^{\mathrm{c}}$ & $91 \pm 5$ & $5569 \pm 75$ & $4306 \pm 96$ \\
\hline
\end{tabular}

Data are presented as the means \pm SEM values of 8 mice per group. LVEDP, left ventricular end-diastolic pressure; LVESP, left ventricular end-systolic pressure; $\mathrm{dP} / \mathrm{dt}$ max, positive-derived pressure; $\mathrm{dP} / \mathrm{dt}$ min, negative-derived pressure; ISO, isoprenaline; CTS, cryptotanshinone; ${ }^{\mathrm{a}} \mathrm{P}<0.05,{ }^{\mathrm{b}} \mathrm{P}<0.01$ vs. control group; ${ }^{\mathrm{c}} \mathrm{P}<0.05$ vs. ISO group.

A

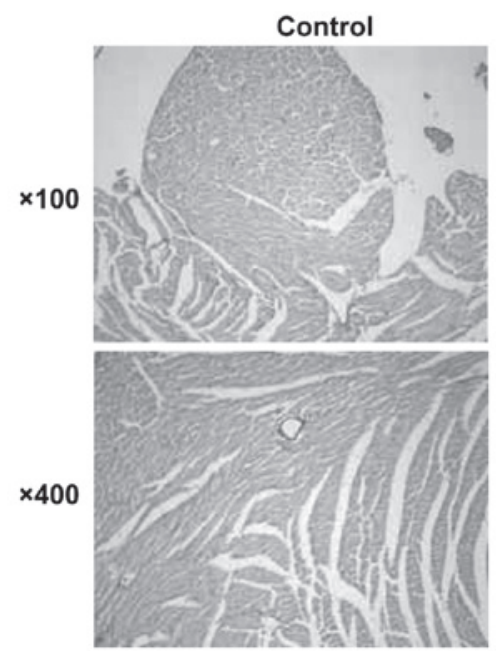

B
ISO
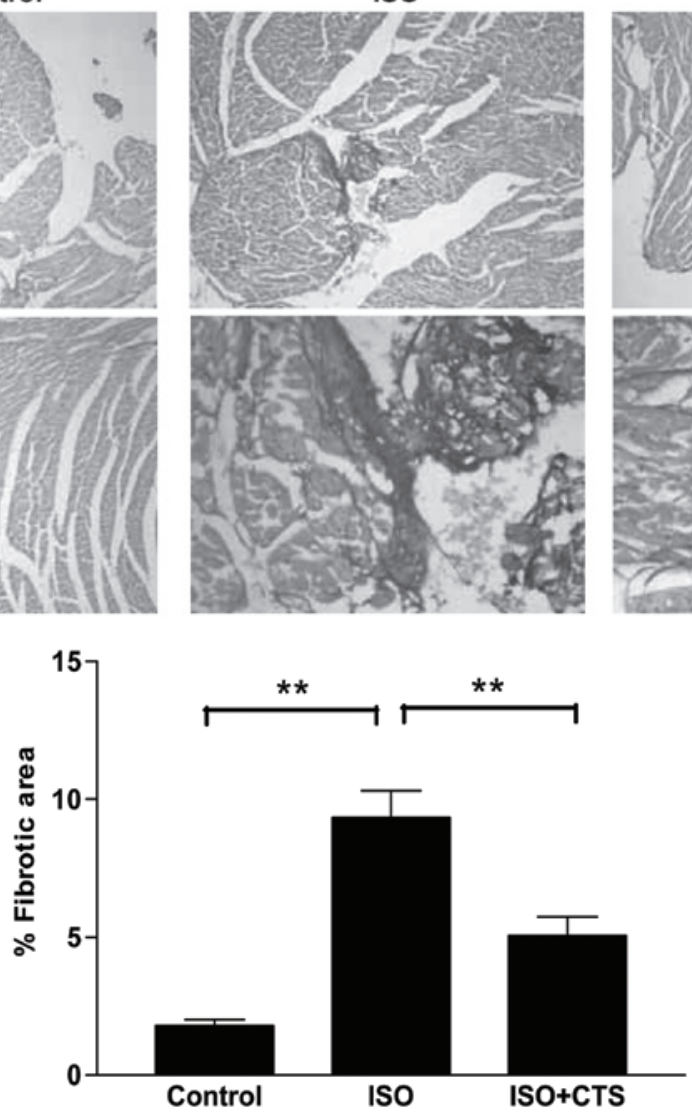

ISO+CTS
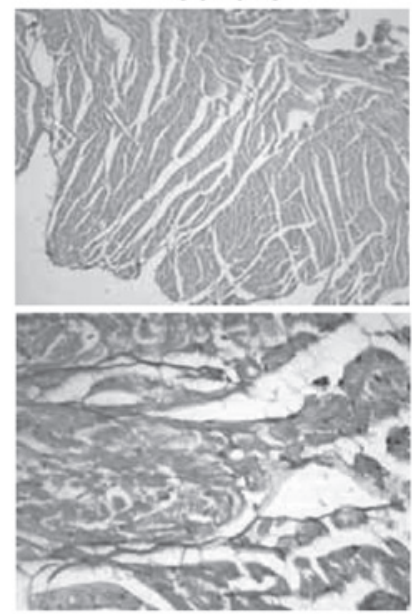

Figure 1. Effect of cryptotanshinone on cardiac fibrosis. (A) Representative photomicrographs of picrosirius red-stained sections of hearts at the level of the papillary muscle; upper panel, original magnification x100; lower panel, original magnification x400. (B) Bar graph showing quantitative analysis of cardiac fibrotic area. ISO, isoprenaline; CTS, cryptotanshinone; data are presented as mean $\pm \mathrm{SEM}$; $\mathrm{n}=5$ mice in each group; ${ }^{* *} \mathrm{P}<0.01$.

\section{Results}

Morphological parameters. The effects of treatment on the morphological parameters of the mice are summarized in Table I. As expected, HW, LVW, HW/BW and LVW/BW were significantly higher in isoprenaline-treated mice compared with the control animals (all $\mathrm{P}<0.01$ ). Notably, the isoprenalineinduced increases in $\mathrm{HW}, \mathrm{LVW}, \mathrm{HW} / \mathrm{BW}$ and $\mathrm{LVW} / \mathrm{BW}$ were markedly attenuated by cryptotanshinone treatment $(\mathrm{P}<0.05$ or $\mathrm{P}<0.01)$.

Hemodynamic parameters. As shown in Table II, isoprenaline treatment elicited a significant increase in the LVEDP $(\mathrm{P}<0.01)$ and a marked decrease in the $\mathrm{dP} / \mathrm{dt} \min (\mathrm{P}<0.05)$. Notably, the isoprenaline-induced elevation in LVEDP was significantly attenuated by the treatment with cryptotanshinone $(\mathrm{P}<0.05)$. By contrast, no significant difference in the LVESP and $\mathrm{dP} / \mathrm{dt}$ max between the treatment groups was found.

Extent of fibrosis. Representative photomicrographs of the heart are shown in Fig. 1A. The extent of fibrosis was significantly increased in the isoprenaline-treated mice $(\mathrm{P}<0.01$; Fig. 1B). Additionally, this increase was partly, but significantly, prevented by cryptotanshinone treatment $(\mathrm{P}<0.01$; Fig. 1B).

Expression and activity of MMP-2 in ventricular myocardium. Compared with the control group, isoprenaline treatment significantly decreased the protein expression and 
A

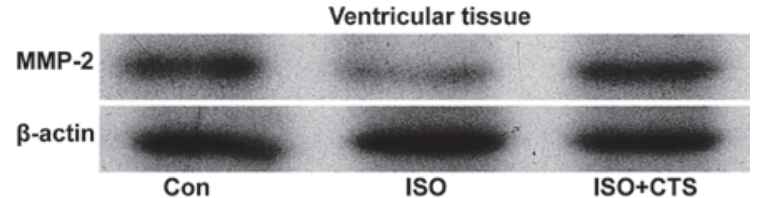

B

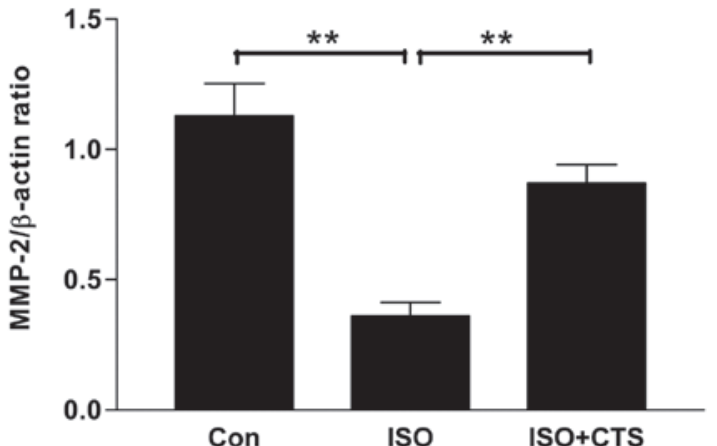

C

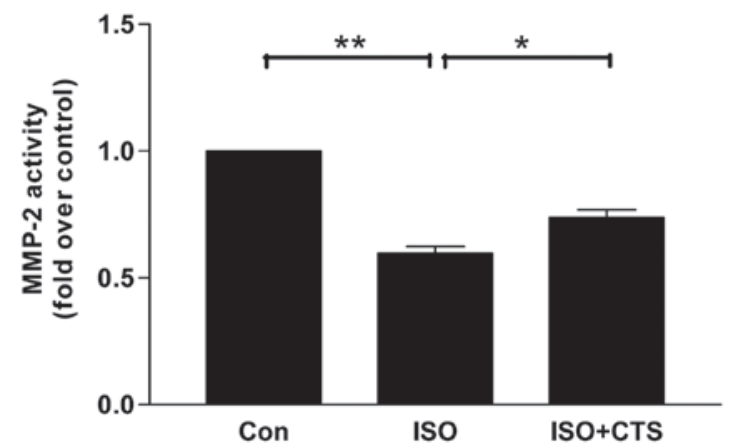

Figure 2. Effects of cryptotanshinone on cardiac MMP-2 protein expression and activity. (A) Representative western blot bands of MMP-2 and $\beta$-actin in the ventricular myocardium from control (Con), Isoprenaline-treated (ISO) and isoprenaline plus cryptotanshinone-treated (ISO+CTS) mice. (B) MMP$2 / \beta$-actin ratio is shown in the bar graph. (C) Bar graph showing quantitative analysis of MMP-2 activity. Data are presented as mean \pm SEM; $n=5$ mice in each group; ${ }^{*} \mathrm{P}<0.05 ;{ }^{* *} \mathrm{P}<0.01$. MMP, matrix metalloproteinase.

degenerative activity of MMP-2 (P<0.01; Fig. 2). Moreover, the inhibitory effects of isoprenaline were markedly attenuated by cryptotanshinone treatment $(\mathrm{P}<0.01$ or $\mathrm{P}<0.05$; Fig. 2).

Expression and activity of MMP-2 in cultured cardiac fibroblasts. In the cultured cardiac fibroblasts, isoprenaline incubation dose-dependently increased the protein expression and degenerative activity of MMP- $2(\mathrm{P}<0.01$ or $\mathrm{P}<0.05$; Fig. 3). Furthermore, the protein expression and degenerative activity of MMP-2 were significantly reduced in the isoprenaline-treated fibroblasts (both $\mathrm{P}<0.01$, Fig. 4) but these decreases were partly prevented by treatment with cryptotanshinone (both $\mathrm{P}<0.05$, Fig. 4).

\section{Discussion}

There are two novel findings in the present study, which used in vitro cultured cardiac fibroblasts and an in vivo mouse model of isoprenaline-induced cardiac fibrosis. First, cryptotanshinone was able to partly, but significantly, prevent the cardiac fibrosis caused by isoprenaline injection. Second, the antifibrotic effect of cryptotanshinone was associated with
$\mathbf{A}$

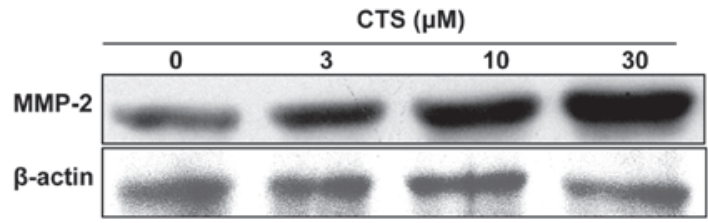

B

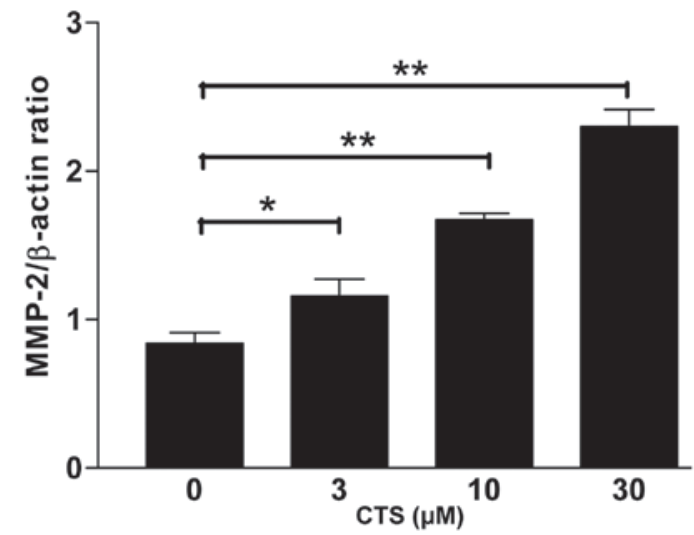

C

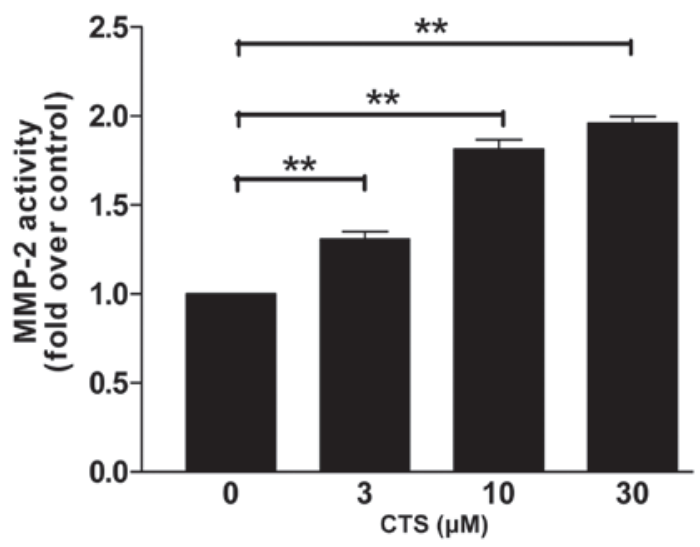

Figure 3. Effects of cryptotanshinone on MMP-2 protein expression and activity in cultured cardiac fibroblasts. (A) Representative western blot bands of MMP-2 and $\beta$-actin in cultured cardiac fibroblasts treated with $0,3,10$ or $30 \mu \mathrm{M}$ cryptotanshinone (CTS) for $24 \mathrm{~h}$. (B) MMP- $2 / \beta$-actin ratio is shown in the bar graph. (C) Bar graph showing quantitative analysis of MMP-2 activity. Data are presented as mean \pm SEM of three independent experiments; ${ }^{*} \mathrm{P}<0.05 ;{ }^{* *} \mathrm{P}<0.01$. MMP, matrix metalloproteinase.

comparable elevations of MMP-2 protein expression and activity. These findings suggest that the downregulation and inactivation of MMP-2 contributes to the isoprenaline-induced cardiac fibrosis and cryptotanshinone-induced attenuation of cardiac fibrosis.

MMP-2, which has collagenolytic activity on types I, II and III collagen, has been implicated in cardiac fibrosis (23). Several lines of evidence have indicated that impaired matrix degradation is associated with reduced MMP-2 activity $(9,10,24)$. However, the results were inconsistent and appeared to depend on the animal model used. Chronic pressure overload in MMP-2-deficient mice is associated with reduced cardiac interstitial fibrosis and MMP-2 transgenic mice have been reported to be associated with increased replacement fibrosis and perivascular fibrosis $(25,26)$. The results of the present study support the theory that the degenerative activity of MMP-2 is markedly impaired in the fibrotic myocardium. Moreover, this phenomenon was also observed in cultured cardiac fibroblasts stimulated with isoprenaline 
A

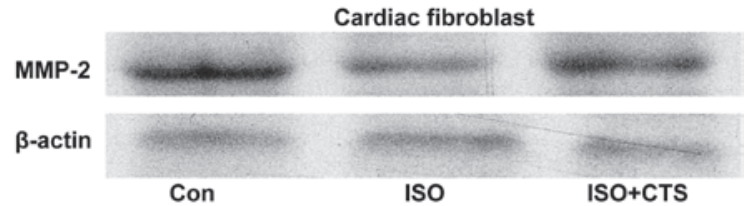

B

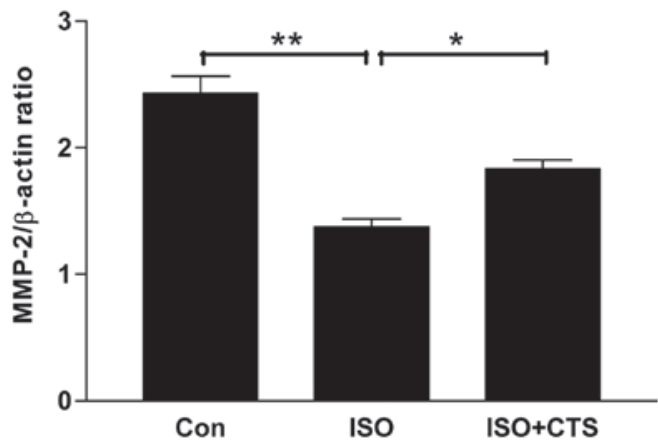

C

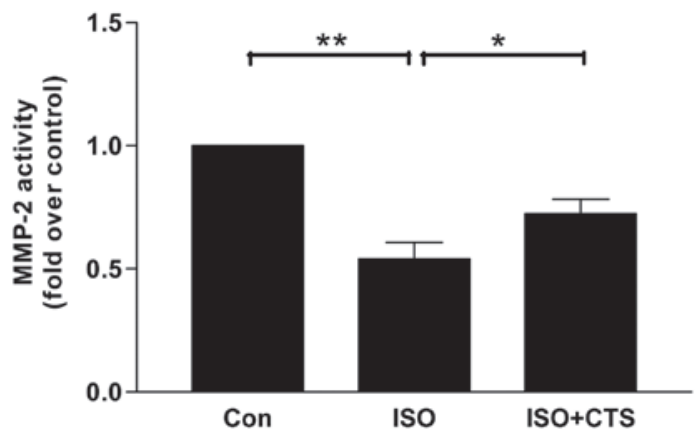

Figure 4. Effects of cryptotanshinone on isoprenaline-induced downregulation and inactivation of MMP-2 in cultured cardiac fibroblasts (A) Representative western blot bands of MMP-2 and $\beta$-actin in cultured cardiac fibroblasts treated with vehicle (Con), isoprenaline (ISO) $(10 \mu \mathrm{M})$ or isoprenaline plus cryptotanshinone (ISO+CTS) $(30 \mu \mathrm{M})$ for $24 \mathrm{~h}$. (B) MMP$2 / \beta$-actin ratio is shown in the bar graph. (C) Bar graph showing quantitative analysis of MMP-2 activity. Data are presented as mean \pm SEM of three independent experiments; ${ }^{*} \mathrm{P}<0.05 ;{ }^{* *} \mathrm{P}<0.01$. MMP, matrix metalloproteinase.

in the present study. Similarly, stimulation with high glucose has also been found to decrease the activity of MMP-2 in rat mesangial cells (27). Collectively, the augmentation of MMP-2 activation may be a useful therapeutic strategy to prevent cardiac fibrosis.

Salvia miltiorrhiza is a well-known herb used in traditional Chinese medicine for the treatment of cardiocerebrovascular diseases. The two main active ingredients extracted from the Salvia miltiorrhiza root are tanshinone and cryptotanshinone, which have distinct biological effects. Although tanshinone has been shown to have an antifibrotic effect in cultured cardiac fibroblasts (14), the role of cryptotanshinone in the development of cardiac fibrosis has not been reported. The present study demonstrated for the first time that treatment with cryptotanshinone is an effective method to prevent isoprenaline-induced cardiac fibrosis. Moreover, our results also suggest that the antifibrotic effect of cryptotanshinone is due to the upregulation and activation of MMP-2. However, other mechanisms cannot be excluded. A previous study demonstrated that cryptotanshinone may inhibit proinflammatory cytokine production, reduce neutrophil infiltration and downregulate adhesion molecules through the inhibition of NF- $\mathrm{NB}$-activation during ischemia and reperfusion and finally attenuate ischemia and reperfusion-induced microcirculatory disturbances (28). Therefore, anti-inflammatory action may also be involved in cryptotanshinone-induced cardiac protection.

The inhibitory effect of cryptotanshinone in isoprenaline-induced increases in HW, LVW, HW/BW and LVW/BW indicated that cryptotanshinone may have antihypertrophic action. The beneficial effect in hemodynamic homeostasis further confirmed this hypothesis. However, this issue warrants further investigation.

In summary, our data reveal that cryptotanshinone is able to upregulate and activate MMP-2 in cultured cardiac fibroblasts and that this effect is able to reverse the isoprenaline-induced downregulation and inactivation of MMP-2 in cardiac fibroblasts. Our data also reveal that the antifibrotic effect of cryptotanshinone is associated with the upregulation and activation of MMP-2 in the ventricular myocardium. Taken together, our findings further support the hypothesis that MMP-2 activation has therapeutic potential against the development of cardiac fibrosis and highlight the mechanisms involved in the antifibrotic effect of cryptotanshinone.

\section{Acknowledgements}

This study was supported by grants from the Medical Scientific Research Program of PLA Chengdu Military Area Command during the 11th Five-Year Plan Period (to D.C. Yang, no. MB09023) and the Science \& Technology Project of Sichuan Province (to D.C. Yang, no. 2010JY0028).

\section{References}

1. Vasan RS and Benjamin EJ: Diastolic heart failure - no time to relax. N Engl J Med 344: 56-59, 2001.

2. Weber KT, Brilla CG and Janicki JS: Myocardial fibrosis: functional significance and regulatory factors. Cardiovasc Res 27: 341-348, 1993.

3. Yamamoto K, Masuyama T, Sakata Y, et al: Myocardial stiffness is determined by ventricular fibrosis, but not by compensatory or excessive hypertrophy in hypertensive heart. Cardiovasc Res 55: 76-82, 2002 .

4. Yamamoto K, Takahashi Y,Mano T, et al: N-methylethanolamine attenuates cardiac fibrosis and improves diastolic function: inhibition of phospholipase D as a possible mechanism. Eur Heart J 25: 1221-1229, 2004

5. Zeisberg EM, Tarnavski O, Zeisberg M, et al: Endothelial-tomesenchymal transition contributes to cardiac fibrosis. Nat Med 13: 952-961, 2007.

6. Janicki JS, Brower GL, Henegar JR and Wang L: Ventricular remodeling in heart failure: the role of myocardial collagen. Adv Exp Med Biol 382: 239-245, 1995.

7. Spinale FG: Matrix metalloproteinases: regulation and dysregulation in the failing heart. Circ Res 90: 520-530, 2002.

8. Li YY, McTiernan CF and Feldman AM: Interplay of matrix metalloproteinases, tissue inhibitors of metalloproteinases and their regulators in cardiac matrix remodeling. Cardiovasc Res 46: 214-224, 2000.

9. Van Linthout S, Seeland U, Riad A, et al: Reduced MMP-2 activity contributes to cardiac fibrosis in experimental diabetic cardiomyopathy. Basic Res Cardiol 103: 319-327, 2008.

10. Lupia E, Elliot SJ, Lenz O, et al: IGF-1 decreases collagen degradation in diabetic NOD mesangial cells: implications for diabetic nephropathy. Diabetes 48: 1638-1644, 1999.

11. Lee RT: Matrix metalloproteinase inhibition and the prevention of heart failure. Trends Cardiovasc Med 11: 202-205, 2001.

12. Ling S, Dai A, Guo Z and Komesaroff PA: A preparation of herbal medicine Salvia miltiorrhiza reduces expression of intercellular adhesion molecule-1 and development of atherosclerosis in apolipoprotein E-deficient mice. J Cardiovasc Pharmacol 51: 38-44, 2008. 
13. Lei XL and Chiou GC: Studies on cardiovascular actions of Salvia miltiorrhiza. Am J Chin Med 14: 26-32, 1986.

14. Maki T, Kawahara Y, Tanonaka K, Yagi A and Takeo S: Effects of tanshinone VI on the hypertrophy of cardiac myocytes and fibrosis of cardiac fibroblasts of neonatal rats. Planta Med 68: 1103-1107, 2002.

15. Yang L, Zou XJ, Gao X, et al: Sodium tanshinone IIA sulfonate attenuates angiotensin II-induced collagen type I expression in cardiac fibroblasts in vitro. Exp Mol Med 41: 508-516, 2009.

16. Lee DS, Lee SH, Noh JG and Hong SD: Antibacterial activities of cryptotanshinone and dihydrotanshinone I from a medicinal herb, Salvia miltiorrhiza Bunge. Biosci Biotechnol Biochem 63 2236-2239, 1999.

17. Chiou WF and Don MJ: Cryptotanshinone inhibits macrophage migration by impeding F-actin polymerization and filopodia extension. Life Sci 81: 109-114, 2007.

18. Mei Z, Zhang F, Tao L, et al: Cryptotanshinone, a compound from Salvia miltiorrhiza modulates amyloid precursor protein metabolism and attenuates beta-amyloid deposition through upregulating alpha-secretase in vivo and in vitro. Neurosci Lett 452: 90-95, 2009.

19. Stempien-Otero A, Plawman A, Meznarich J, Dyamenahalli T, Otsuka G and Dichek DA: Mechanisms of cardiac fibrosis induced by urokinase plasminogen activator. J Biol Chem 281: 15345-15351, 2006.

20. Kacimi R, Vessey DA, Honbo N and Karliner JS: Adult cardiac fibroblasts null for sphingosine kinase-1 exhibit growth dysregulation and an enhanced proinflammatory response. J Mol Cell Cardiol 43: 85-91, 2007.

21. Yang D, Ma S, Li D, Tang B and Yang Y: Angiotensin II receptor blockade improves matrix metalloproteinases/tissue inhibitor of matrix metalloproteinase-1 balance and restores fibronectin expression in rat infarcted myocardium. Biochem Biophys Res Commun 388: 606-611, 2009.
22. Riches K, Morley ME, Turner NA, et al: Chronic hypoxia inhibits MMP-2 activation and cellular invasion in human cardiac myofibroblasts. J Mol Cell Cardiol 47: 391-399, 2009.

23. Aimes RT and Quigley JP: Matrix metalloproteinase-2 is an interstitial collagenase. Inhibitor-free enzyme catalyzes the cleavage of collagen fibrils and soluble native type I collagen generating the specific 3/4- and 1/4-length fragments. J Biol Chem 270: 5872-5876, 1995.

24. Han SY, Jee YH, Han KH, et al: An imbalance between matrix metalloproteinase-2 and tissue inhibitor of matrix metalloproteinase- 2 contributes to the development of early diabetic nephropathy. Nephrol Dial Transplant 21: 2406-2416, 2006.

25. Bergman MR, Teerlink JR, Mahimkar R, et al: Cardiac matrix metalloproteinase-2 expression independently induces marked ventricular remodeling and systolic dysfunction. Am J Physiol Heart Circ Physiol 292: H1847-1860, 2007.

26. Matsusaka H, Ide T, Matsushima S, et al: Targeted deletion of matrix metalloproteinase 2 ameliorates myocardial remodeling in mice with chronic pressure overload. Hypertension 47: 711-717, 2006.

27. Singh R, Song RH, Alavi N, Pegoraro AA, Singh AK and Leehey DJ: High glucose decreases matrix metalloproteinase-2 activity in rat mesangial cells via transforming growth factorbeta1. Exp Nephrol 9: 249-257, 2001.

28. Jin YC, Kim CW, Kim YM, et al: Cryptotanshinone, a lipophilic compound of Salvia miltiorrriza root, inhibits TNF-alphainduced expression of adhesion molecules in HUVEC and attenuates rat myocardial ischemia/reperfusion injury in vivo. Eur J Pharmacol 614: 91-97, 2009. 